\title{
Pressure Ramp Testing for Optimization of End-Expiratory Pressure Settings in Adaptive Servo-Ventilation Therapy
}

\author{
Masakazu Hori, MD; Teruhiko Imamura, MD, PhD; \\ Nikhil Narang, MD; Koichiro Kinugawa, MD, PhD
}

\begin{abstract}
Background: Clinical outcomes of adaptive servo-ventilation (ASV) therapy have not been rigorously assessed. Optimal device settings ascertained by a pressure ramp test may increase the utility of ASV therapy.
\end{abstract}

\begin{abstract}
Methods and Results: Patients with congestive heart failure (CHF) who underwent ASV therapy were prospectively included in the study. Patients in the ramp test group underwent a pressure ramp test, during which the end-expiratory pressure was optimized to maximize cardiac output (assessed using the AESCLONE mini). The control group consisted of age-matched patients who received ASV therapy with a default pressure $5 \mathrm{cmH}_{2} \mathrm{O}$. The primary endpoint was a composite of all-cause death and heart failure recurrence, and was compared between the 2 groups. Of a total of 37 patients, 11 each were included in the ramp test and control groups. Median patient age was 73 years (interquartile range 59-75 years) and 16 were men. There were no significant differences in baseline characteristics between the 2 groups. In the ramp test group, end-expiratory pressure was optimized between 2 and $5 \mathrm{cmH} \mathrm{H}_{2} \mathrm{O}$ in each patient. The 2-year incidence of the primary endpoint tended to be lower in the ramp test than control group ( $0 \%$ vs. 59\%; $P=0.080)$.
\end{abstract}

Conclusions: Pressure ramp testing may be a promising strategy to optimize device pressure settings in patients with $\mathrm{CHF}$ undergoing ASV therapy. Larger-scale trials are needed to validate our findings.

Key Words: Heart failure; Hemodynamics; Non-invasive positive pressure support

A daptive servo-ventilation (ASV; AutoSet-CS; ResMed, Sydney, NSW, Australia) is non-invasive positive pressure ventilation therapy that stabilizes respiratory mechanics, suppresses sympathetic nervous system activity, and reduces pulmonary and systemic congestion by decreasing preload and afterload in patients with congestive heart failure $(\mathrm{CHF})$, regardless of the presence of sleep-disordered breathing. ${ }^{1}$ As a result, cardiac output increases, facilitating reverse remodeling and allowing for improved clinical outcomes over time. ${ }^{2}$

However, in the SERVE-HF trial, ASV therapy was associated with an increased risk of cardiovascular death compared with the control arm. ${ }^{3}$ Given these results, careful patient selection and clinical follow-up are highly encouraged to avoid therapy-related adverse events. In the SERVE-HF trial, the end-expiratory pressure was relatively high to aggressively suppress any coexisting sleep-disordered breathing. An inappropriately high pressure setting may reduce cardiac output, and thus increase the risk of worse clinical outcomes. ${ }^{4}$ We hypothesized that optimizing the device pressure setting may improve the efficacy of ASV therapy in patients with clinical heart failure, preventing low cardiac output. ${ }^{1}$

We previously proposed a "pressure ramp test", during which hemodynamics are invasively measured at each endexpiratory pressure to determine an ideal device pressure setting. ${ }^{5}$ One of the limitations of the pressure ramp test was the requirement for invasive hemodynamic assessment, which is challenging to perform routinely. The AESCLONE mini (Osypla Medical, Berlin, Germany) is a non-invasive device that can estimate cardiac output. ${ }^{6}$ Taking all this into consideration, we hypothesized that ASV therapy will improve clinical outcomes if the pressure setting is optimized through the pressure ramp test using the AESCLONE mini, during which the set end-expiratory pressure is titrated to a level where cardiac output is maximized. In the present study, we compared clinical outcomes between patients receiving ASV therapy with an optimized pressure setting and those receiving ASV therapy with a default pressure setting.

Received September 28, 2021; revised manuscript received October 14, 2021; accepted October 19, 2021; J-STAGE Advance Publication released online November 18, 2021 Time for primary review: 13 days

Second Department of Internal Medicine, Toyama University, Toyama (M.H., T.I., K.K.), Japan; Advocate Christ Medical Center, Oak Lawn, IL (N.N.), USA

K.K. is a member of Circulation Reports' Editorial Team.

The first two authors contributed equally to this study (M.H., T.I.).

Mailing address: Teruhiko Imamura, MD, PhD, FESC, FAHA, FACC, FHFSA, FAPSC, FACP, FICA, FASA, FJCC, Second Department of Internal Medicine, Toyama University, 2630 Sugitani, Toyama 930-0194, Japan. E-mail: teimamu@med. u-toyama.ac.jp

All rights are reserved to the Japanese Circulation Society. For permissions, please e-mail: cr@j-circ.or.jp

ISSN-2434-0790 


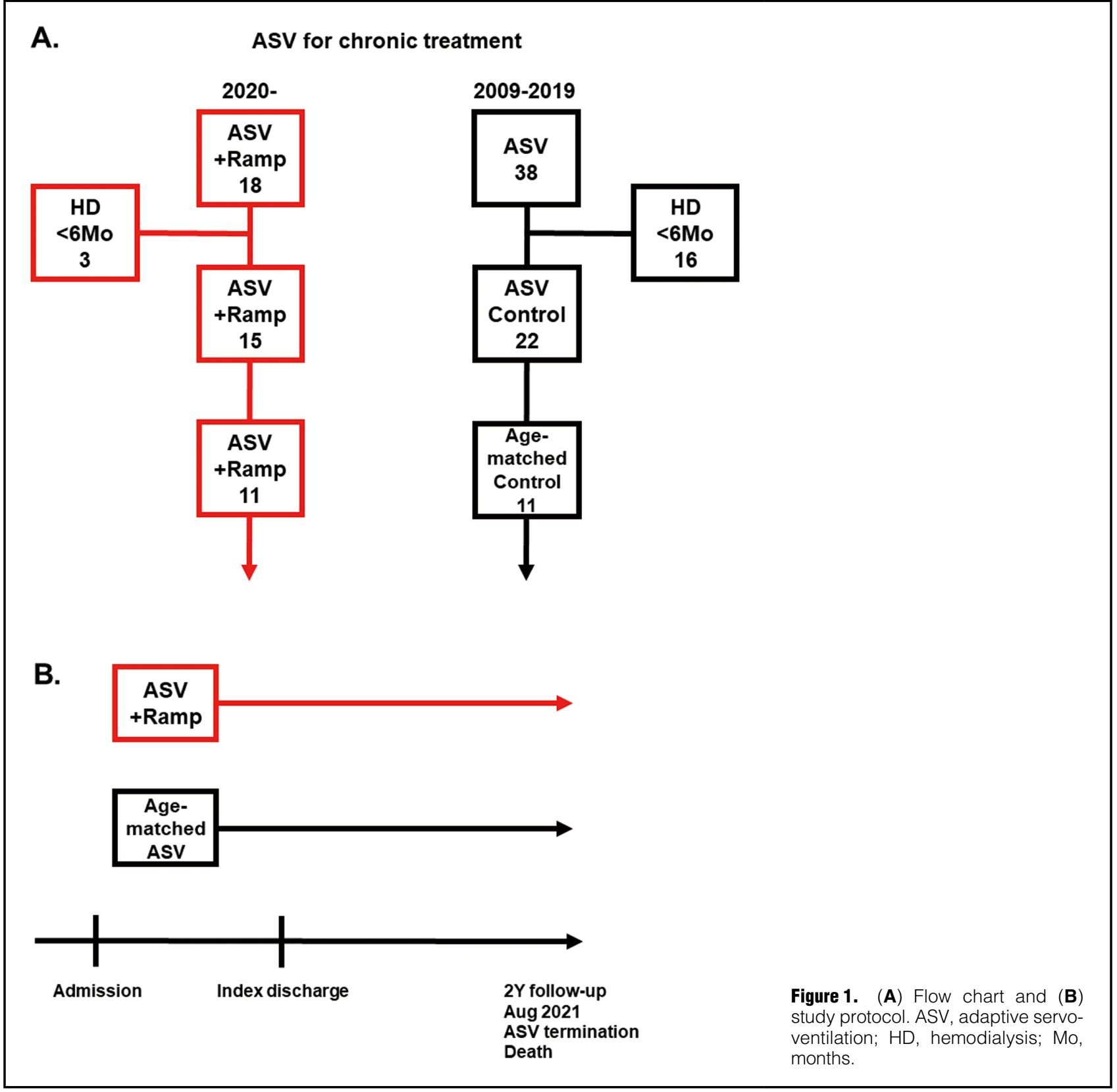

\section{Methods}

\section{Patient Selection}

Patients with CHF who continued ASV therapy with default pressure setting as described below for $>6$ months at our institute between 2009 and 2019 were retrospectively included in this analysis as candidates for inclusion in the control group.

From 2020, the protocol at University of Toyama was updated, and we started using ASV pressure ramp tests to optimize pressure settings as detailed below. Patients who underwent the ASV pressure ramp tests and then continued ASV therapy with an optimized pressure setting for $>6$ months between 2020 and 2021 were prospectively considered for inclusion in this study as part of the ramp test group.

The indication for ASV therapy was at the discretion of the attending physicians. Briefly, to prevent heart failure recurrence, ASV therapy was considered in chronic heart failure patients with symptomatic systemic and/or pulmonary congestion, assessed using different modalities, including physical examination, chest X-ray, and transthoracic echocardiography. Patients who underwent ASV therapy as an acute treatment, those who discontinued ASV therapy within 6 months, and those who did not tolerate ASV therapy due to discomfort during the initial trials were excluded from the study. Patients who were dependent on hemodiafiltration for end-stage renal disease were also excluded.

This study was approved by the Clinical Research Review Board, University of Toyama (IRB no. R2015154). The need for written informed consent was waived because of the observational nature of this study, and an opt-out approach 


\begin{tabular}{|c|c|c|c|c|}
\hline & $\begin{array}{c}\text { Total cohort } \\
(n=22)\end{array}$ & $\begin{array}{l}\text { Ramp test group } \\
(n=11)\end{array}$ & $\begin{array}{c}\text { Control group } \\
(n=11)\end{array}$ & $P$ value \\
\hline \multicolumn{5}{|l|}{ Demographics } \\
\hline Age (years) & 73 [59-75] & 74 [64-77] & $71[62-75]$ & 0.52 \\
\hline Male sex & $16(73)$ & $8(73)$ & $8(73)$ & 1.0 \\
\hline $\mathrm{BMI}\left(\mathrm{kg} / \mathrm{m}^{2}\right)$ & $20.8[19.2-23.5]$ & $19.8[19.0-23.5]$ & $21.0[19.6-23.3]$ & 0.75 \\
\hline Ischemic etiology & 7 (32) & $4(36)$ & $3(27)$ & 0.65 \\
\hline \multicolumn{5}{|l|}{ Comorbidity } \\
\hline Hypertension & $12(55)$ & $7(64)$ & $5(45)$ & 0.39 \\
\hline Diabetes & $8(36)$ & $3(27)$ & $5(45)$ & 0.38 \\
\hline Chronic kidney disease & $13(59)$ & $8(73)$ & $5(45)$ & 0.19 \\
\hline History of stroke & $5(23)$ & $2(18)$ & $3(27)$ & 0.61 \\
\hline Atrial fibrillation & $7(32)$ & $2(18)$ & $5(45)$ & 0.17 \\
\hline History of HF admission & $11(50)$ & $4(36)$ & $7(64)$ & 0.20 \\
\hline \multicolumn{5}{|l|}{ Echocardiography } \\
\hline LVEDd $(\mathrm{mm})$ & 55 [49-66] & 54 [49-64] & $55[51-68]$ & 0.56 \\
\hline LVEF (\%) & 43 [33-59] & 47 [37-62] & 41 [32-49] & 0.40 \\
\hline LVEF $>40 \%$ & $12(50)$ & $6(50)$ & $6(50)$ & 0.67 \\
\hline LA diameter $(\mathrm{mm})$ & $43[40-51]$ & $43[40-49]$ & $43[41-50]$ & 0.70 \\
\hline Moderate or greater MR & $9(41)$ & $6(55)$ & $3(27)$ & 0.19 \\
\hline Moderate or greater TR & 7 (32) & $5(45)$ & $2(18)$ & 0.17 \\
\hline \multicolumn{5}{|l|}{ Medication } \\
\hline$\beta$-blocker & $14(64)$ & $7(64)$ & $7(64)$ & 1.0 \\
\hline RAS inhibitor & $15(68)$ & $8(73)$ & $7(64)$ & 0.65 \\
\hline MRA & $9(41)$ & $5(45)$ & $4(36)$ & 0.67 \\
\hline Diuretics & $13(59)$ & $6(55)$ & $7(64)$ & 0.67 \\
\hline
\end{tabular}

Continuous variables are presented as the median [interquartile range], whereas categorical variables are presented as $n(\%)$. Continuous variables were compared by the Mann-Whitney $U$ test; categorical variables were compared by Fischer's exact test. BMI, body mass index; HF, heart failure; LA, left atrial; LVEDd, left ventricular end-diastolic diameter; LVEF, left ventricular ejection fraction; MR, mitral regurgitation; MRA, mineralocorticoid receptor antagonist; RAS, renin-angiotensin system; TR, tricuspid regurgitation.

was used.

\section{ASV Therapy}

We used an advanced bi-level positive airway pressure unit, ASV, together with a fitted full-face mask. The device analyzes breathing patterns automatically and provides pressure support, which is synchronized with breathing via logic algorithms. The device was set to deliver $5 \mathrm{cmH}_{2} \mathrm{O}$ positive end-expiratory pressure (default pressure setting) and suitable minimum-maximum inspiratory support between 3 and $10 \mathrm{cmH}_{2} \mathrm{O}$. ASV therapy was performed for over $4 \mathrm{~h}$ every night. ASV therapy was terminated at the discretion of the investigators when congestion was deemed to have improved.

\section{ASV Pressure Ramp Test}

All patients assigned to the ramp test group underwent ASV pressure ramp titration testing. Briefly, following a detailed explanation of the concept and mechanism of the device and mask fitting tests, cardiac output was estimated using AESCLONE mini at $5 \mathrm{cmH}_{2} \mathrm{O}$ expiratory positive airway pressure (the default pressure setting). Similar measurements were performed at each incremental expiratory positive airway pressure setting from 1 to $10 \mathrm{cmH}_{2} \mathrm{O}$, or up to the tolerable pressure. Finally, a pressure setting associated with maximum cardiac output was selected as the fixed pressure setting.

\section{Clinical Data}

Baseline characteristics, including demographics, medications, and laboratory, and echocardiographic data at the time of ASV initiation, were assessed. For the echocardiographic assessment, left ventricular ejection fraction was calculated by the modified Simpson's method. For the ramp test group, estimated cardiac output data during the pressure ramp tests were also obtained.

The observation period was terminated at the end of a 2-year follow-up period, the time of data retrieval (August 2021), the time of ASV termination, or the time of death. The primary endpoint was a composite of all-cause death or heart failure readmissions that required intravenous diuretics during in-hospital observation. The heart failure recurrence rate was also assessed.

At 3-12 months after the initiation of ASV therapy, echocardiographic data were analyzed, if available. Changes in echocardiographic data between baseline and follow-up were compared in each group as a secondary outcome.

\section{Statistical Analyses}

Continuous variables are expressed as the median and interquartile range (IQR) and were compared between the groups using the Mann-Whitney U test. Categorical variables are expressed as numbers and percentages and were compared between groups using Fischer's exact test. Propensity score matching analysis was performed to establish 


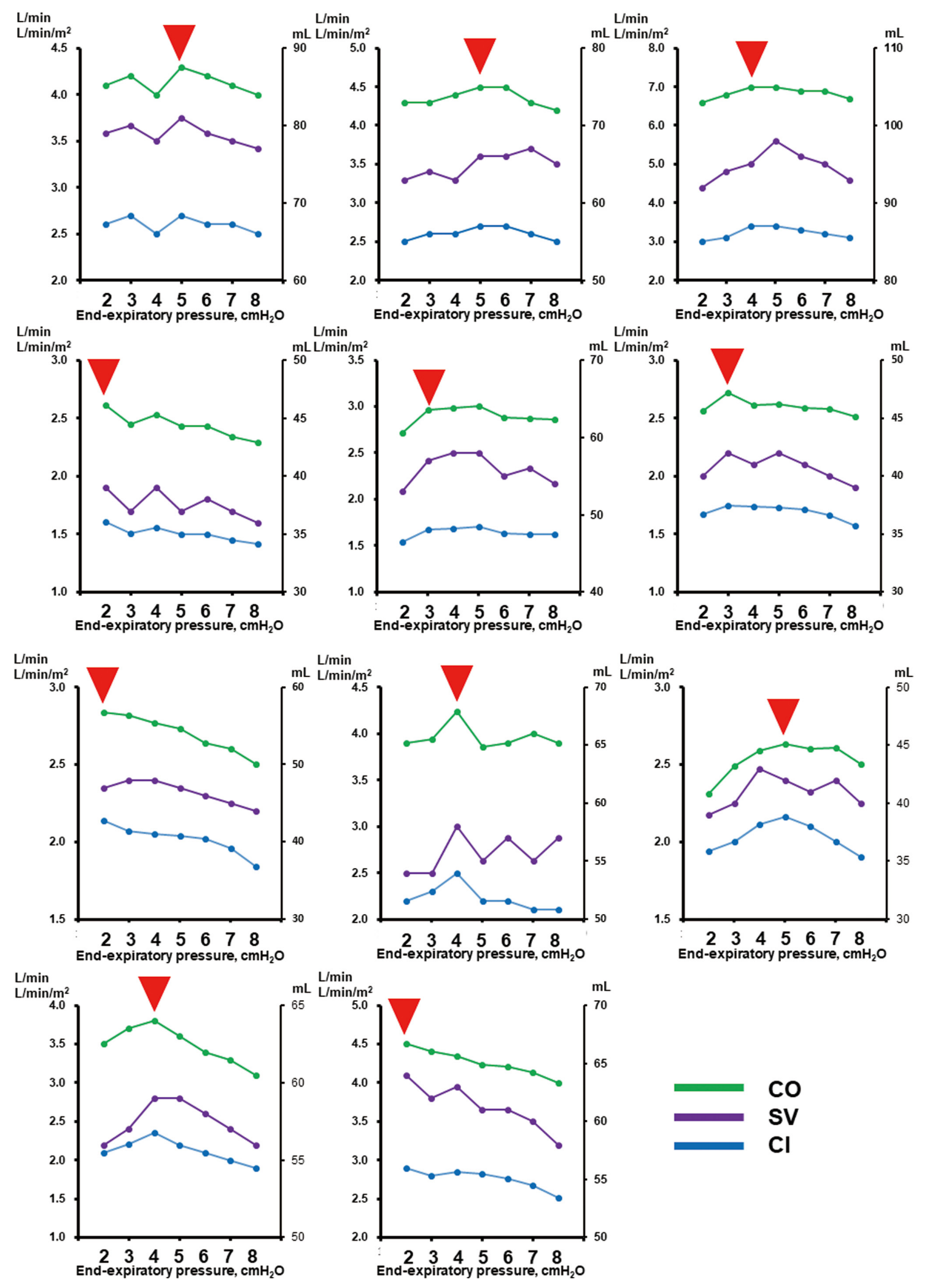

Figure 2. Changes in hemodynamic parameters during the pressure ramp test in 11 individual patients. Given the findings, the end-expiratory pressure was set at the pressure indicated by the arrowhead for each patient. $\mathrm{Cl}$, cardiac index; $\mathrm{CO}$, cardiac output; SV, stroke volume. 


\begin{tabular}{|c|c|c|c|c|}
\hline & $\begin{array}{l}\text { Total } \\
(n=22)\end{array}$ & $\begin{array}{l}\text { Ramp test group } \\
(n=11)\end{array}$ & $\begin{array}{c}\text { Control group } \\
(n=11)\end{array}$ & $P$ value \\
\hline \multicolumn{5}{|l|}{ Laboratory data } \\
\hline Hemoglobin (g/dL) & $11.8[10.5-13.0]$ & $12.5[9.2-13.1]$ & $11.8[11.1-13.0]$ & 0.80 \\
\hline Serum albumin (g/dL) & $3.5[3.3-3.7]$ & $3.4[3.2-3.7]$ & $3.7[3.4-4.0]$ & 0.30 \\
\hline Serum sodium (mEq/L) & $139[137-140]$ & 139 [137-141] & 138 [137-140] & 1.0 \\
\hline eGFR $\left(\mathrm{mL} / \mathrm{min} / 1.73 \mathrm{~m}^{2}\right)$ & $45.7[33.1-55.2]$ & $42.0[32.9-50.9]$ & $50.6[45.2-54.5]$ & 0.48 \\
\hline Plasma BNP (pg/mL) & 249 [144-395] & 322 [154-402] & 240 [144-369] & 0.70 \\
\hline \multicolumn{5}{|l|}{ Medication } \\
\hline$\beta$-blocker & $17(77)$ & $9(82)$ & $8(73)$ & 0.61 \\
\hline RAS inhibitor & $20(91)$ & $11(100)$ & $9(82)$ & 0.14 \\
\hline MRA & $13(59)$ & $7(64)$ & $6(55)$ & 0.67 \\
\hline Diuretics & $17(77)$ & $9(82)$ & $8(73)$ & 0.61 \\
\hline
\end{tabular}

Continuous variables are presented as the median [interquartile range] and categorical variables are presented as $\mathrm{n}(\%)$. Continuous variables were compared by the Mann-Whitney $U$ test; categorical variables are compared by Fischer's exact test. BNP, B-type natriuretic peptide; eGFR, estimated glomerular filtration rate. Other abbreviations as in Table 1.

\begin{tabular}{|c|c|c|c|}
\hline & Baseline & Follow-up & $P$ value \\
\hline \multicolumn{4}{|l|}{ Ramp test group $(n=9)$} \\
\hline LVEDd (mm) & 58 [54-68] & $53[51-58]$ & 0.069 \\
\hline LVEF (\%) & 47 [37-59] & 57 [44-65] & 0.092 \\
\hline LA diameter (mm) & $43[41-50]$ & $41[32-46]$ & $0.043^{*}$ \\
\hline Moderate or greater MR & $6(67)$ & $3(33)$ & 0.25 \\
\hline Moderate or greater TR & $5(56)$ & $1(11)$ & 0.13 \\
\hline Plasma BNP (pg/mL) & 322 [154-402] & $125[76-215]$ & $0.035^{\star}$ \\
\hline \multicolumn{4}{|l|}{ Control group $(n=8)$} \\
\hline LVEDd (mm) & $55[50-68]$ & $60[49-72]$ & 0.36 \\
\hline LVEF (\%) & 37 [32-53] & 34 [25-52] & 0.11 \\
\hline LA diameter (mm) & $44[41-50]$ & $45[40-50]$ & 0.83 \\
\hline Moderate or greater MR & $3(38)$ & $2(25)$ & 1.0 \\
\hline Moderate or greater TR & $2(25)$ & $1(13)$ & 1.0 \\
\hline Plasma BNP (pg/mL) & $240[144-369]$ & 215 [154-303] & 0.25 \\
\hline
\end{tabular}

Continuous variables are presented as the median [interquartile range] and categorical variables are presented as $\mathrm{n}(\%)$. Continuous variables were compared by the Mann-Whitney $U$ test; categorical variables were compared by the Wilcoxon signed-rank test or the McNemar test. ${ }^{*} P<0.05$. Abbreviations as in Tables 1,2 .

a $1: 1$ age-matched cohort between the ramp test and control groups with a caliper window of 0.3 .

The cumulative incidence of the primary outcomes was compared between the 2 groups using the log-rank test. Intragroup trends in continuous variables were assessed using the Wilcoxon signed-rank test, whereas trends in categorical variables were assessed using the McNemar test.

All statistical analyses were performed using the SPSS 22 (IBM Corp., Armonk, NY, USA). Two-tailed $\mathrm{P}<0.05$ was considered significant.

\section{Results}

\section{Baseline Characteristics}

From 2009 to 2019, 38 patients received ASV therapy as chronic treatment; 16 of these patients were excluded from the study because they were dependent on hemodialysis or because the duration of ASV therapy was $<6$ months.
From 2020, 18 patients received ASV therapy after a ramp test; 3 of these patients were excluded for the same reasons (Figure 1A).

Of a total of 15 patients in the ramp test group and 22 in the control group, 11 patients in the ramp test group and 11 age-matched patients in the control group were included in this study (Figure 1B). The characteristics of the 22 patients in this study are provided in Table 1. The median patient age was 73 years (IQR 59-75 years) and 73\% were men. The median left ventricular ejection fraction was $43 \%$ (IQR 33-59\%). Seven patients (32\%) had ischemic cardiomyopathy, $9(41 \%)$ had moderate or greater mitral regurgitation, and $7(32 \%)$ had moderate or greater tricuspid regurgitation (Table 1).

\section{ASV Pressure Ramp Tests}

Trends in cardiac output, cardiac index, and stroke volume in each of the 11 patients who underwent a pressure ramp 


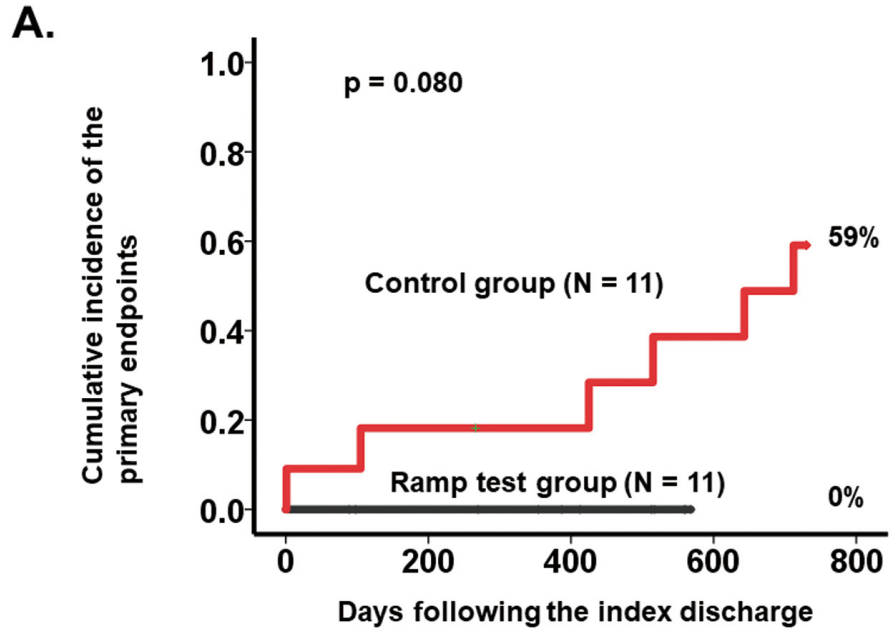

Ramp test group: 11 Control group: 11

B.

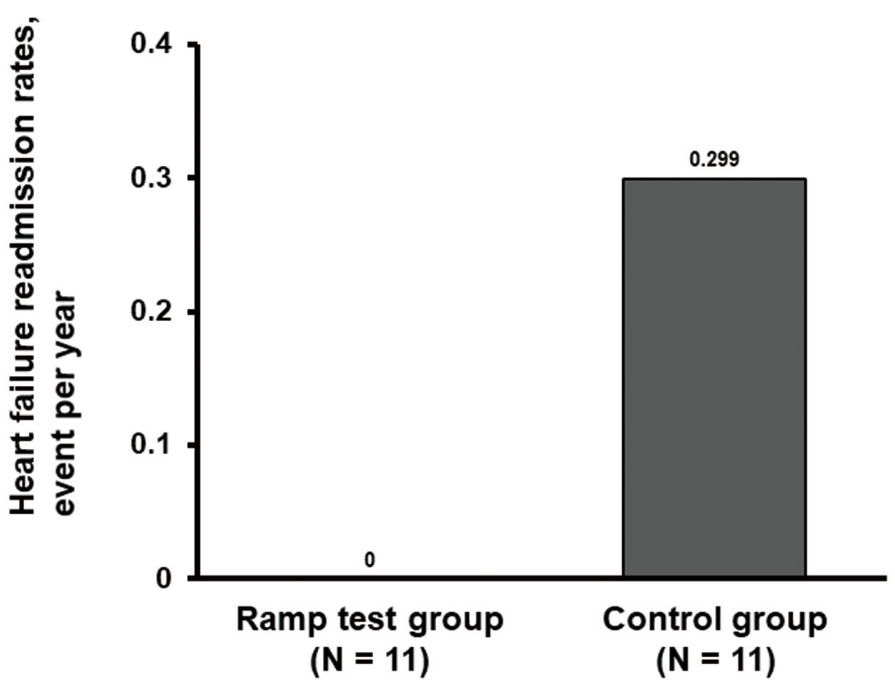

Figure 3. (A) Cumulative incidence of the primary endpoint and (B) heart failure event rates in the ramp test and control groups. test are shown in Figure 2. The trend curves at incremental pressure settings are unique in each patient. As a whole, peak of cardiac output (Figure 2, arrowheads) was located at a relatively lower pressure setting than the default setting of $5 \mathrm{cmH}_{2} \mathrm{O}$. In all cases, cardiac output gradually decreased at incremental pressure settings beyond the peak pressure. Consequently, the end-expiratory pressure was set at $2 \mathrm{cmH}_{2} \mathrm{O}$ in 3 patients, at $3 \mathrm{cmH}_{2} \mathrm{O}$ in 2 patients, at $4 \mathrm{cmH}_{2} \mathrm{O}$ in 3 patients, and at $5 \mathrm{cmH}_{2} \mathrm{O}$ in 3 patients. The set pressure remained unchanged during the observation period.

\section{Clinical Data at Index Discharge}

Laboratory data, including plasma B-type natriuretic peptide (BNP) and medications, did not differ significantly between the 2 groups at index discharge ( $\mathrm{P}>0.05$ for all; Table 2$)$.

\section{Trends in Clinical Parameters}

Follow-up echocardiography was performed a median of
233 days (IQR 164-301 days) following the index discharge (Table 3). Data were lacking for several patients because of unexpected death or transfer to another institute. In the ramp test group, left heart size decreased and ejection fraction increased. The prevalence of moderate or greater valve diseases tended to decrease. In the control group, these parameters remained unchanged relatively to baseline ( $\mathrm{P}>0.05$ for all). Plasma BNP concentrations decreased significantly in the ramp test group $(\mathrm{P}=0.035)$, but were unchanged in the control group $(\mathrm{P}=0.25)$.

\section{Clinical Outcomes}

ASV therapy was continued for a median of 712 days (IQR 346-730 days) in the control group and 413 days (IQR 312-538 days) in the ramp test group. ASV was used for a median of $5.7 \mathrm{~h} /$ day (IQR $5.1-6.5 \mathrm{~h} /$ day) in the control group and $6.3 \mathrm{~h} /$ day (IQR $5.4-6.9 \mathrm{~h} /$ day) in the ramp test group $(\mathrm{P}<0.001)$. ASV therapy was terminated in 6 patients in the control group due to poor adherence, with 
$<4 \mathrm{~h}$ ASV use per night, whereas ASV therapy was continued in all patients in the ramp test group.

During the observation period (median 515 days [IQR 270-712 days]), 3 patients died, 2 patients had heart failure readmissions, and 1 patient had both heart failure and died in the control group; in contrast, none of these events was observed in the ramp test group. The cumulative incidence of the primary outcomes tended to be lower in the ramp test group ( $0 \%$ vs. $59 \%, \mathrm{P}=0.080$; Figure 3A).

In the control group, 2 patients had multiple heart failure readmissions, and the event rate was 0.299 events/year. In the ramp test group, the event rate was zero (Figure 3B).

\section{Subanalyses}

There were 12 patients with preserved ejection fraction $(>40 \%)$. Among these patients, the cumulative incidence of the primary endpoint was $0 \%$ in the ramp test group $(n=6)$, compared with $40 \%$ in the control group $(n=6 ; \mathrm{P}=0.50)$. Among 10 patients with reduced ejection fraction $(<40 \%)$, the cumulative incidence of the primary endpoint was $0 \%$ in the ramp test group $(\mathrm{n}=5)$, compared with $80 \%$ in the control group $(\mathrm{n}=5 ; \mathrm{P}=0.068)$.

\section{Power Analysis}

Given the finding of the cumulative incidence of the primary endpoint ( $0 \%$ vs. $60 \%$ ), an $\alpha$ error of 0.05 , and a sample size of 22, the power value of this analysis was calculated to be 0.47 .

\section{Discussion}

In this study we investigated the clinical implication of device pressure ramp testing and optimization of the endexpiratory pressure setting during ASV therapy by comparing outcomes with age-matched patients receiving ASV therapy at default settings. In the ramp test group, the optimal end-expiratory pressure was set between 2 and $5 \mathrm{cmH}_{2} \mathrm{O}$, whereas it was set at $5 \mathrm{cmH}_{2} \mathrm{O}$ (default setting) in the control group. The ramp test group had a greater incidence of reverse remodeling, in addition to lower morbidity and mortality.

\section{Pressure Ramp Test}

One of the primary components of successful ASV therapy is an increase in cardiac output, which may lead to downstream improvements in cardiac structure and function, in addition to lower heart failure readmission event rates. ${ }^{1}$ ASV therapy with appropriate pressure settings should reduce both preload and afterload while increasing cardiac output. In the SERVE-HF trial, a relatively higher endexpiratory pressure setting to treat sleep disorder may have led to decreases in cardiac output, and subsequently higher rates of major adverse events. ${ }^{\mathbf{3}, 4}$ This is why we proposed a pressure ramp test, during which the optimal pressure is elucidated by measuring the hemodynamics at each pressure setting.

In this study we proposed a modified and simplified protocol using the AESCLONE mini to estimate cardiac output non-invasively during pressure ramp testing. ${ }^{6} \mathrm{Com}-$ pared with the previous test protocol, ${ }^{5}$ this modified protocol should be more generalizable and easier to implement. In the present study, cardiac output was maximized at a relatively lower pressure than the default setting of $5 \mathrm{cmH}_{2} \mathrm{O}$. As expected, cardiac output tended to decrease at a pressure higher than the default setting. Some patients may experience discomfort at high pressure settings. This discomfort, along with lower cardiac output, may stimulate a sympathetic nerve response, thus further attenuating the odds of improvement in cardiac reverse remodeling while increasing the risk of worsening clinical heart failure. ${ }^{7}$

\section{Ramp Test and Clinical Outcome}

Patients who underwent the ramp test achieved a greater degree of cardiac reverse remodeling, demonstrated by a decrease in left ventricular size and an increase in left ventricular ejection fraction. ${ }^{8}$ Continuous cardiac unloading with an increase in cardiac output, along with suppression of sympathetic nerve activity, during ASV therapy with an optimized pressure setting underscores the benefit of this therapy.

All patients who underwent the ramp test continued with ASV therapy, whereas half the patients in the control group discontinued ASV therapy. The duration of ASV was shorter in the control group. In some patients, a default pressure setting without any pressure adjustment may be too strong and cause discomfort, resulting in poor adherence and subsequently poor clinical outcomes. By providing favorably optimized pressure support, the ramp test may improve adherence to ASV therapy, which is quite important for the clinical benefit of this therapy to be realized.

In some patients who underwent the ramp test, the final pressure setting did not change from the default setting. However, we cannot predict the optimal pressure without a ramp test, and we strongly encourage clinicians to perform a ramp test before initiating ASV therapy. Given the findings of the subanalyses, in which ASV therapy at the default pressure setting was associated with worse outcomes, particularly among those with a reduced ejection fraction, a ramp test should be particularly encouraged for such patients.

\section{Study Limitations}

This was a single-center study with a small cohort. This study was a proof-of-concept study, and further large multicenter studies should be conducted to validate our findings. We cannot rule out the presence of selection bias. Clinicians did their best to select appropriate patients for ASV therapy, using chest X-ray, echocardiography, and physical examination, as well as patient symptomology, to obtain evidence of systemic/pulmonary congestion. ${ }^{1}$ ASV therapy was performed for the management of the chronic phase of CHF and our findings cannot be extrapolated to treatment of the acute phase. Given the small sample size, we used only age for background matching. We attempted to match more variables but could not obtain an appropriate cohort size. Although there were no statistically significant differences in baseline characteristics between the 2 groups, we cannot rule out the effects of potential uninvestigated confounders. The pressure setting was fixed during the observation period. The implications of repeated ramp tests should be investigated in further studies. We did not collect data regarding the existence of sleep disorder, and ASV therapy was performed to treat congestion. Thus, the applicability of our findings to patients with sleep disorder remains unknown. Finally, we did not include heart failure patients without ASV therapy as a comparator group in this study because the focus of the study was the add-on impact of the ramp test beyond conventional ASV therapy using the default pressure setting. 


\section{Conclusions}

Pressure ramp testing may be a promising strategy to optimize device pressure setting in patients undergoing ASV therapy. A larger-scale randomized control trial should be performed to validate our findings.

\section{Acknowledgment}

T.I. receives grant support from JSPS KAKENHI (JP20K17143).

\section{Sources of Funding}

This study did not receive any specific funding.

\section{Disclosures}

K.K. is a member of Circulation Reports' Editorial Board. The remaining authors have no conflicts of interest to declare.

\section{IRB Information}

This study was approved by the Clinical Research Review Board, University of Toyama (IRB no. R2015154).

\section{References}

1. Imamura T, Kinugawa K. What is the optimal strategy for adap- tive servo-ventilation therapy? Int Heart J 2018; 59: 683-688.

2. Momomura S, Seino Y, Kihara Y, Adachi H, Yasumura Y, Yokoyama H, et al. Adaptive servo-ventilation therapy for patients with chronic heart failure in a confirmatory, multicenter, randomized, controlled study. Circ J 2015; 79: 981-990.

3. Cowie MR, Woehrle H, Wegscheider K, Angermann C, d'Ortho MP, Erdmann E, et al. Adaptive servo-ventilation for central sleep apnea in systolic heart failure. $N$ Engl J Med 2015; 373: 10951105.

4. Kihara Y, Seino Y, Momomura S; SAVIOR-C Investigators. Adaptive servo-ventilation for central sleep apnea in heart failure. $N$ Engl J Med 2016; 374: 687-688.

5. Imamura T, Nitta D, Kinugawa K. Optimization of pressure settings during adaptive servo-ventilation support using real-time heart rate variability assessment: Initial case report. BMC Cardiovasc Disord 2017; 17: 11.

6. Nakayama A, Iwama K, Makise N, Domoto Y, Ishida J, Morita $\mathrm{H}$, et al. Use of a non-invasive cardiac output measurement in a patient with low-output dilated cardiomyopathy. Intern Med 2020; 59: $1525-1530$

7. Koyama T, Watanabe H, Tamura Y, Oguma Y, Kosaka T, Ito H. Adaptive servo-ventilation therapy improves cardiac sympathetic nerve activity in patients with heart failure. Eur J Heart Fail 2013; 15: 902-909.

8. Haruki N, Takeuchi M, Kaku K, Yoshitani H, Kuwaki H, Tamura M, et al. Comparison of acute and chronic impact of adaptive servo-ventilation on left chamber geometry and function in patients with chronic heart failure. Eur J Heart Fail 2011; 13: $1140-1146$. 